\title{
Stößt die Frauenquote an ihre Grenzen? Eine Untersuchung der Bundestagswahl 2009
}

\section{Louise K. Davidson-Schmich und Isabelle Kürschner}

\section{Der Einfluss der Parteien auf die Kandidatenauswahl}

Die Bundesrepublik wird aufgrund ihres Wahl- und Parteiensystems und des relativ hohen Anteils von Frauen in den Parlamenten häufig als Beispiel für die Einführung, Umsetzung und Auswirkung von Quotenregelungen herangezogen. Dabei ist der Einfluss der Parteien auf die Kandidatenauswahl in Praxis und Forschung unbestritten. Parteien haben in Deutschland den verfassungsgemäßen Auftrag inne, die aktive Teilnahme der Bürger am politischen Leben zu fördern und zur Übernahme öffentlicher Verantwortung befähigte Bürger heranzubilden. ${ }^{1}$ Somit liegt die angemessene Beteiligung von Frauen in der Politik in erster Linie in der Hand der Parteien. Die Ansprache von Frauen und die entsprechende Förderung weiblicher Kandidaten bis zum Bekleiden politischer Ämter und Mandate gehören zu den Aufgaben, die eine Parteiorganisation zu erfüllen hat. Dennoch war die innerparteiliche Unterrepräsentation von Frauen und ihre damit einhergehende politische Machtlosigkeit für die Parteien in Deutschland lange Zeit kein Thema. Erst als mit dem Aufkommen der Frauenbewegung in den 1980er Jahren Teile der weiblichen Bevölkerung ihren Unmut über die herrschenden Zustände artikulierten, begannen die Parteien über Strategien zur gezielten Förderung von Frauen nachzudenken. Obwohl sich beim Ziel einer angemessenen Vertretung der weiblichen Bevölkerung - alle Parteien einig waren, ergriffen sie unterschiedliche Maßnahmen, um es zu erreichen.

Diese Maßnahmen weisen eine gewisse Ähnlichkeit mit den skandinavischen Ländern auf, wo man ein Zusammenspiel von Parteiquoten und Parteienwettbewerb beobachten kann. Modellhaft kann dies folgendermaßen dargestellt werden ${ }^{2}$ : Eine kleine Partei im linken Spektrum führt eine Frauenquote ein, erzielt gute Wahlergebnisse und setzt damit die nächste, meist größere Partei, die stärker zur Mitte tendiert, unter Zugzwang. Diese reagiert aus Angst vor Wahlverlusten und führt ebenfalls eine Frauenquote ein, was wiederum einen gewissen Anpassungsdruck auf die nächste Partei ausübt. Durch diesen Domino-Effekt können kleine Parteien entscheidenden Einfluss auf alle Parteien und somit auf den Frauenanteil im gesamten Parlament ausüben. Selbst Parteien, die sich weiterhin der Einführung einer Frauenquote verweigern, stehen letztlich unter Druck, Frauen stärker zu fördern, um die Unterschiede in der Repräsentanz nicht zu augenscheinlich werden zu lassen.

1 Vgl. Gesetz über die politischen Parteien (Parteiengesetz) in der Fassung der Bekanntmachung vom 31. Januar 1994 (BGBl. I, S. 149), zuletzt geändert durch Art. 2 des Gesetzes vom 22. Dezember 2004 (BGBl. I, S. 3673).

2 Vgl. Heinrich-Böll-Stiftung (Hrsg.), Unterrepräsentanz von Frauen in der Kommunalpolitik, Vorläufiger Abschlussbericht des Forschungsprojekts „Frauenunterrepräsentanz in der Kommunalpolitik“, Berlin 2009, S. 15. 


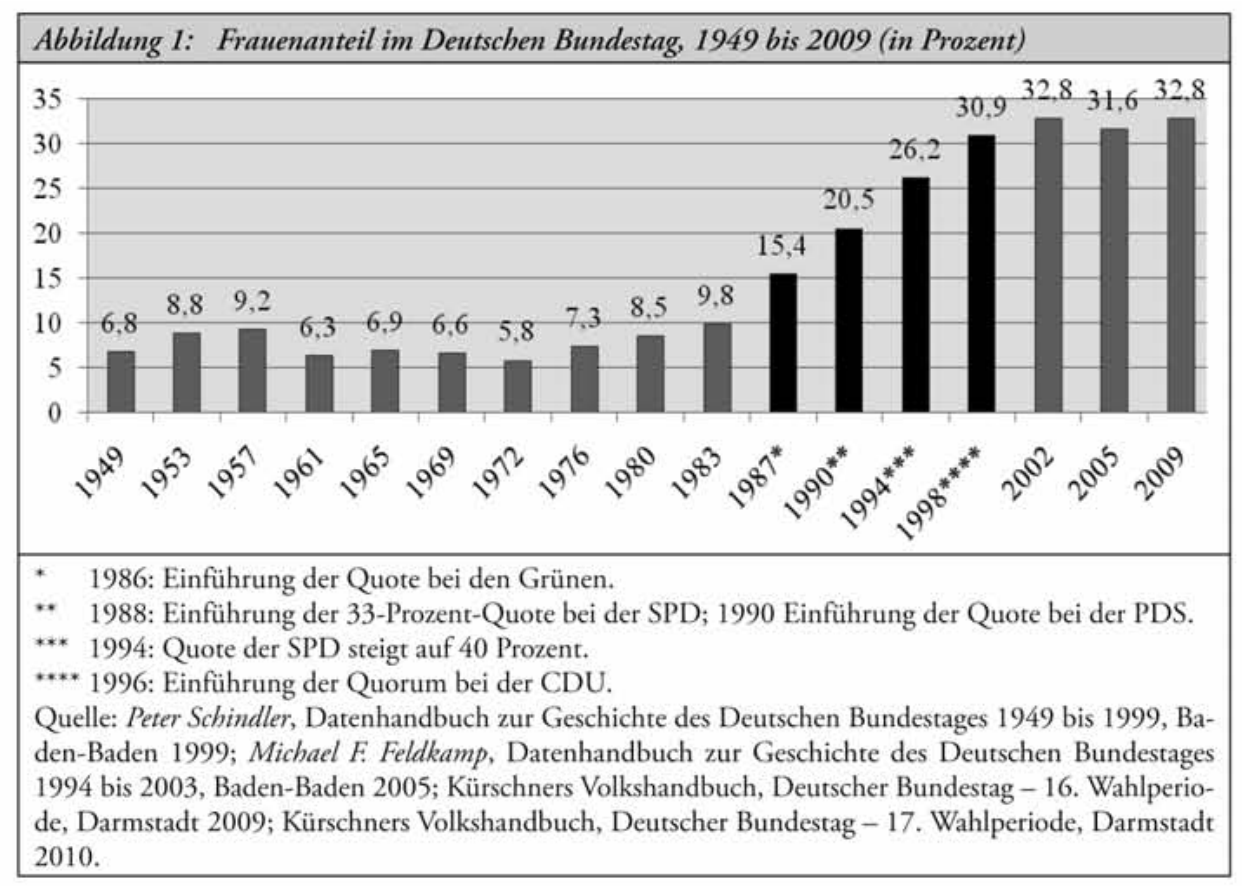

An dieser Abbildung lässt sich das Zusammenspiel von Parteiquoten und Parteienwettbewerb sehr gut erkennen: Mit dem Einzug der Grünen, der ersten Partei mit Frauenquote, in den Deutschen Bundestag stieg der Frauenanteil erstmals in der Geschichte des Parlaments auf über zehn Prozent. Die SPD führte - aus Angst vor dem Verlust weiblicher Wählerstimmen - 1988 ebenfalls eine Frauenquote ein, und der Frauenanteil wuchs um weitere fünf Prozentpunkte. 1996 beschloss die CDU die Einführung des Quorums von 33, Prozent und die SPD steigerte ihre Quote von 33 auf 40 Prozent. Bei der darauffolgenden Wahl 1998 erhöhte sich der Frauenanteil im Bundestag erstmals über 30 Prozent. Auf diesem - im weltweiten Vergleich hohen Niveau - stagniert er jedoch seit nunmehr einer Dekade, obwohl der Frauenanteil in der Bevölkerung bei 51 Prozent liegt. ${ }^{3}$

\section{Einführung der ersten Frauenquote}

Als erste Partei beschlossen die Grünen bereits bei ihrer Gründung 1979 eine Frauenquote, nach der mindestens die Hälfte aller Ämter und Mandate mit Frauen und Wahllisten alternierend mit Männern und Frauen zu besetzen sind. Mit ihrem Einzug in den Bundestag kam es zu einer deutlichen Zunahme von Frauen im Parlament. Der ersten Grünen-Fraktion (1983 bis 1987) gehörten zehn Frauen und 18 Männer an, der zweiten (1987 bis 1990) bereits 25 Frauen und 19 Männer. Der dadurch auftretende Wettbewerb, vor allem um

3 Nach Angaben des Statistischen Bundesamtes lebten Ende 2008 41,8 Millionen Frauen in Deutschland. Das entspricht einem Anteil an der Bevölkerung von 51 Prozent. 
junge Wählerinnen, veranlasste die SPD, ebenfalls eine Frauenquote einzuführen. Sie beschloss 1988 eine 33-Prozent-Quote für beide Geschlechter für Ämter und Mandate und steigerte diese ab 1998 auf 40 Prozent. Die Konkurrenzsituation zwischen SPD und Grünen führte in den 1980er Jahren zu einem starken Anstieg des Frauenanteils im Bundestag: von 9,8 Prozent im Jahr 1983 auf 20,5 Prozent 1990. Einen Beitrag dazu leistete auch die CDU, die im Dezember 1994, eine Quote mit einem Anteil von einem Drittel plante, was bei der Abstimmung auf dem Parteitag aber zunächst scheiterte. Stattdessen beschloss sie 1996 ein sogenanntes Frauenquorum, demzufolge Frauen und Männer zu mindestens einem Drittel an Parteiämtern und öffentlichen Mandaten beteiligt sein sollen. ${ }^{4}$ Zwar hat das Quorum lediglich Empfehlungscharakter und ist entsprechend leicht zu umgehen, doch demonstrierte die CDU damit zumindest eine gewisse Offenheit gegenüber der Beteiligung von Frauen. Die CSU hat auf ihrem Parteitag 2010 beschlossen, eine Frauenquote von 40 Prozent für Vorstände auf Bezirks- und Landesebene einzuführen. Auf Wahllisten und Direktmandate hat dies jedoch keinen unmittelbaren Einfluss. Die Linke verpflichtete sich, in Vorstände, Kommissionen, Arbeitsgremien und Delegiertenversammlungen mindestens zur Hälfte Frauen zu wählen. ${ }^{5}$ Damit bleibt die FDP die letzte im Bundestag vertretene Partei, die sich bis heute gegen die Einführung einer festen Frauenquote ausspricht. Sie setzt weiterhin auf unverbindliche Empfehlungen und strebt eine „gleichwertige Repräsentanz von Frauen und Männern in allen Gremien der Gesellschaft" ${ }^{\text {"6 an. }}$

In Tabelle 1 ist sehr gut zu erkennen, welchen Einfluss die Einführung der Frauenquote auf die einzelnen Fraktionen hatte. Bis zum Einzug der Grünen in den Bundestag 1983 lag der Frauenanteil in allen Fraktionen unter oder nur knapp über zehn Prozent. Eine Wahlperiode später (1987), erhöhte sich der Frauenanteil bei der SPD immerhin um fast sechs Punkte, obwohl die Partei selbst seinerzeit noch keine Quote eingeführt hatte. Dieses Phänomen kann als Nachahmungseffekt betrachtet werden, das heißt eine Partei - die SPD versuchte, das Erfolgsrezept einer anderen - der Grünen - zu kopieren. Ein leichter Nachahmungseffekt war in jenem Jahr auch bei der FDP zu beobachten, deren Frauenanteil immerhin um fast vier Prozentpunkte wuchs. CDU und CSU blieben hiervon vorerst unberührt. Nach der Einführung der Frauenquote bei der SPD im Jahr 1988 stieg deren Frauenanteil in der darauffolgenden Wahlperiode (1990) um weitere elf Punkte an. Bei den drei übrigen Parteien, CDU, CSU und FDP, waren diesmal starke Nachahmungseffekte sichtbar: Die FDP erhöhte ihren Frauenanteil um fast acht, die CDU um sechs und die CSU um immerhin vier Prozentpunkte. Die Einführung des Frauenquorums in der CDU und die Erhöhung der Quote von 33 auf 40 Prozent in der SPD bewirkte bei der Wahl 1998 eine weitere Steigerung bei allen Parteien, so dass in der 14. Wahlperiode fast 31 Prozent Frauen dem Bundestag angehörten. Seither ist jedoch - nach dem zunächst rapiden Anstieg - überall eine Abflachung oder sogar ein leichter Rückgang des Frauenanteils fest-

4 Diese Vorgabe ist jedoch in einem zweiten Wahlgang zu umgehen: „Wird bei Gruppenwahlen zu Parteiämtern von der Kreisverbandsebene an aufwärts in einem ersten Wahlgang das Frauenquorum von einem Drittel nicht erreicht, ist dieser Wahlgang ungültig. Es ist ein zweiter Wahlgang vorzunehmen, zu dem weitere Vorschläge gemacht werden können. Dessen Ergebnis ist unabhängig von dem dann erreichten Frauenanteil gültig." Satzung der CDU, Abschnitt C, $\$ 15$ Gleichstellung von Frauen und Männern, Berlin 2007.

5 Vgl. Bundessatzung der Partei Die Linke, $\$ 10$ Geschlechterdemokratie, Rostock 2010.

6 Wiesbadener Grundsätze der FDP, III. Die liberale Bürgergesellschaft im demokratischen Bürgerstaat, Wiesbaden 1997. 


\begin{tabular}{|c|c|c|c|c|c|c|c|}
\hline Wahlperiode & $\mathrm{CDU}$ & $\mathrm{CSU}$ & FDP & SPD & Grüne & Linke & Anmerkungen \\
\hline 10. 1983-1987 & 7 & 6 & 9 & 10 & 36 & - & Einzug der Grünen \\
\hline 11. 1987-1990 & 8 & 6 & 13 & 16 & 57 & - & \\
\hline 12. 1990-1994 & 14 & 10 & 20 & 27 & 38 & 47 & $\begin{array}{l}\text { 1. Wahlperiode } \\
\text { nach Einführung der } \\
\text { 33-Prozent-Quote } \\
\text { bei der SPD }\end{array}$ \\
\hline 13. $1994-1998$ & 14 & 6 & 17 & 34 & 59 & 43 & \\
\hline 14. $1998-2002$ & 18 & 13 & 21 & 35 & 57 & 58 & $\begin{array}{l}\text { 1. Wahlperiode nach } \\
\text { Einführung des Quo- } \\
\text { rums bei der CDU } \\
\text { sowie der } 40 \text {-Prozent- } \\
\text { Quote bei der SPD }\end{array}$ \\
\hline 15. 2002-2005 & 23 & 22 & 21 & 38 & 58 & - & \\
\hline 16. $2005-2009$ & 20 & 17 & 25 & 36 & 57 & 46 & \\
\hline 17. 2009- & 22 & 13 & 23 & 38 & 54 & 53 & \\
\hline \multicolumn{8}{|c|}{$\begin{array}{l}\text { Quelle: Peter Schindler, Datenhandbuch zur Geschichte des Deutschen Bundestages } 1949 \text { bis 1999, Ba- } \\
\text { den-Baden 1999; Michael F. Feldkamp, Datenhandbuch zur Geschichte des Deutschen Bundestages } \\
1994 \text { bis 2003, Baden-Baden 2005; Kürschners Volkshandbuch, Deutscher Bundestag - 16. Wahlperio- } \\
\text { de, Darmstadt 2009; Kürschners Volkshandbuch, Deutscher Bundestag - 17. Wahlperiode, Darmstadt } \\
2010 .\end{array}$} \\
\hline
\end{tabular}

zustellen, so dass heute, zwölf Jahre später, weiterhin nur knapp ein Drittel der Mitglieder des Bundestages weiblich sind.

\section{Wirkung der Frauenquote}

Ob eine Frauenquote tatsächlich Wirkung entfalten kann oder nur als symbolische Geste $\mathrm{zu}$ verstehen ist, hängt von verschiedenen Faktoren $\mathrm{ab}^{7}$ : der Mindestanforderung, der Rangfolge und den Sanktionen. Die Mindestanforderung bestimmt, welchen Anteil an Frauen die entsprechende Quote fordert. Weltweit schwanken diese Anteile zwischen fünf (Nepal) und 50 Prozent (zum Beispiel Frankreich), in Deutschland zwischen 33 (CDU) und 50 Prozent (Grüne, Linke). Ziel ist, eine so genannte kritische Masse ${ }^{8}$ von Frauen in Gremien und Parlamenten zu etablieren. Die Rangfolge legt vor allem bei Listenaufstellun-

7 Vgl. Drude Dahlerup / Lenita Freidenvall, Quotas as a ,Fast Track' to Equal Representation for Women: Why Scandinavia Is No Longer the Model, in: Mona Lena Krook / Sarah Childs (Hrsg.), Women, Gender, and Politics: A Reader, Oxford 2010, S. $175-181$.

8 Der Begriff kritische Masse stammt ursprünglich aus der Kernenergie und bezeichnet die für eine Kettenreaktion nötige Mindestmasse spaltbarer Stoffe. In den Sozialwissenschaften hält man eine kritische Masse von Angehörigen einer Minderheit in einer Gruppierung für notwendig, um die Interessen jener Minderheit innerhalb der Gemeinschaft angemessen vertreten zu können. So geht beispielsweise die Europäische Kommission davon aus, dass die kritische Masse von Frauen in einem Parlament 30 Prozent beträgt. Vgl. Europäische Kommission, Frauen und Männer in Entscheidungsprozessen 2007, Analyse der Lage und Entwicklung, Luxemburg 2008. 


\begin{tabular}{|c|c|c|c|c|}
\hline Partei & Quotenregelung laut Satzung & 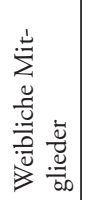 & 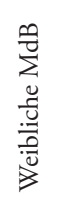 & 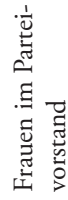 \\
\hline $\mathrm{CDU}$ & $\begin{array}{l}\text { „Frauen sollen an Parteiämtern in der CDU und an öffentlichen Mandaten } \\
\text { mindestens zu einem Drittel beteiligt sein. (...) Wahlgremien können } \\
\text { Kandidatenvorschläge zurückweisen, die Frauen nur unzureichend berück- } \\
\text { sichtigen. Wird bei Gruppenwahlen zu Parteiämtern von der Kreisver- } \\
\text { bandsebene an aufwärts in einem ersten Wahlgang das Frauenquorum von } \\
\text { einem Drittel nicht erreicht, ist dieser Wahlgang ungültig. Es ist ein zwei- } \\
\text { ter Wahlgang vorzunehmen, zu dem weitere Vorschläge gemacht werden } \\
\text { können. Dessen Ergebnis ist unabhängig von dem dann erreichten Frauen- } \\
\text { anteil gültig." }\end{array}$ & 26 & 21 & 28 \\
\hline CSU & „Bei allen Wahlen sind Frauen zu berücksichtigen.“ & 19 & 13 & 26 \\
\hline SPD & $\begin{array}{l}\text { „Wahlvorschläge müssen die satzungsmäßigen Voraussetzungen erfüllen. } \\
\text { Die Personalvorschläge der Vorstände müssen Frauen und Männer mindes- } \\
\text { tens zu je } 40 \text { Prozent berücksichtigen. (...) Um zu erreichen, dass Männer } \\
\text { und Frauen zu mindestens je } 40 \text { Prozent in den Parlamenten und kommu- } \\
\text { nalen Vertretungskörperschaften vertreten sind, werden auf allen Organisa- } \\
\text { tionsebenen satzungsmäßige Vorkehrungen getroffen; (...) Für die Wahl } \\
\text { zum Deutschen Bundestag wird die angemessene Vertretung von Frauen } \\
\text { und Männern durch die Aufstellung der Landeslisten gesichert. Die Auf- } \\
\text { stellung der Landeslisten erfolgt alternierend; eine Frau, ein Mann, begin- } \\
\text { nend mit dem Spitzenkandidaten oder der Spitzenkandidatin, jeder } \\
\text { 5. Platz kann entweder mit einer Frau oder einem Mann besetzt werden.“ } \\
\text { (Ebenso Europäisches Parlament) }\end{array}$ & 31 & 38 & 40 \\
\hline FDP & $\begin{array}{l}\text { Keine Erwähnung in der Satzung. Im Grundsatzprogramm: „Die } \\
\text { gleichgewichtige Repräsentanz von Männern und Frauen in allen Gremien } \\
\text { der Gesellschaft ist anzustreben." }\end{array}$ & 23 & 26 & 20 \\
\hline $\begin{array}{l}\text { Grü- } \\
\text { ne }\end{array}$ & $\begin{array}{l}\text { „Wahllisten sind grundsätzlich alternierend mit Frauen und Männern zu } \\
\text { besetzen, wobei den Frauen die ungeraden Plätze zur Verfügung stehen } \\
\text { (Mindestparität). Frauen können auch auf den geraden Plätzen kandidie- } \\
\text { ren. Reine Frauenlisten sind möglich. Sollte keine Frau für einen Frauen } \\
\text { zustehenden Platz kandidieren bzw. gewählt werden, entscheidet die Wahl- } \\
\text { versammlung über das weitere Verfahren. Die Frauen der Wahlversamm- } \\
\text { lung haben diesbezüglich ein Vetorecht.“ }\end{array}$ & 37 & 54 & 56 \\
\hline Linke & $\begin{array}{l}\text { „Bei Wahlen von Vorständen, Kommissionen, Arbeitsgremien und Dele- } \\
\text { gierten sind grundsätzlich mindestens zur Hälfte Frauen zu wählen. Ist dies } \\
\text { nicht möglich, bleiben die den Frauen vorbehaltenen Mandate unbesetzt, } \\
\text { eine Nachwahl ist jederzeit möglich. Kreis- und Ortsverbände, deren Frau- } \\
\text { enanteil bei weniger als einem Viertel liegt, können im Einzelfall Ausnah- } \\
\text { men beschließen." }\end{array}$ & 38 & 54 & 45 \\
\hline \multicolumn{5}{|c|}{$\begin{array}{l}\text { Quelle: Quotenregelung CDU: Satzung der CDU, a.a.O. (Fn. 4); CSU: Satzung der CSU 2006, } \\
\text { 5. Abschnitt Verfahrensordnung, } \$ 53 \text { Verfahren für alle Wahlen (4). Der im Oktober } 2010 \text { gefasste Be- } \\
\text { schluss, eine 40-Prozent-Quote für Vorstände auf Bezirks- und Landesebene einzuführen, ist noch nicht } \\
\text { in der Satzung verankert; SPD: Wahlordnung der SPD 2005, } \$ 4 \text {, Verfahren bei Kandidatenaufstellung; } \\
\text { FDP: Wiesbadener Grundsätze der FDP, a.a.O. (Fn. 6), S. 11; Grüne: Satzung von Bündnis } 90 \text { / Die } \\
\text { Grünen, o.J. Frauenstatut, 1. Rahmenbedingungen, } \$ 1 \text { Mindestquotierung; Die Linke: Bundessatzung } \\
\text { der Partei Die Linke, a.a.O. (Fn. 5). Weibliche Mitglieder: Bundeszentrale für politische Bildung, Dossier } \\
\text { Parteien. Weibliche MdB: Bundestagsabgeordnete der 17. Wahlperiode, www.bundestag.de (Stand vom } \\
\text { August 2010). Frauen im Parteivorstand: Eigene Berechnung nach Auskunft der jeweiligen Parteien. }\end{array}$} \\
\hline
\end{tabular}


gen fest, an welcher Stelle Frauen repräsentiert sein müssen. Selbst bei einem Frauenanteil von 30 Prozent auf einer Parteiliste ist nämlich noch nicht sicher gestellt, dass auch eine entsprechende Anzahl von ihnen gewählt wird. Dies wird erst durch bestimmte Festlegungen ermöglicht, zum Beispiel, dass in jedem Dreier-, Fünfer- oder Zehnerblock eine gewisse Anzahl von Frauen vertreten sein oder dass gar das Reißverschlussprinzip angewandt werden muss, nach dem Frauen und Männer auf der Liste alternieren. Auch bei der Besetzung von Vorständen und Gremien ist darauf zu achten, dass sich Frauen bereits in den engeren Vorständen, also unter den Vorsitzenden, stellvertretenden Vorsitzenden, Schatzmeistern und Schriftführern befinden und nicht in erster Linie bei den Beisitzern. Schließlich werden sämtliche Vorgaben aber nur dann zuverlässig umgesetzt, wenn entsprechende Sanktionen zur Verfügung stehen, die ein Nichtbefolgen der Vorgaben bestrafen.

Die Sanktionsmöglichkeiten beschränken sich in der Bundesrepublik auf parteiinterne Strafmechanismen, denn im Gegensatz zu anderen Ländern gibt es hier lediglich freiwillige Selbstverpflichtungen von Seiten der Parteien und keine Gesetze, die Frauen den Zugang zu Wahlämtern und -mandaten garantieren. Frankreich und Spanien verfügen hingegen über staatliche Sanktionsmöglichkeiten wie Kürzungen bei der Wahlkampfmittelerstattung oder Ablehnung von Kandidatenlisten bei Nichteinhaltung der vorgegebenen Frauenanteile. ${ }^{9}$ Die Wirksamkeit entsprechender Sanktionsmöglichkeiten zeigt ein vergleichender Blick auf die Frauenanteile der Parteien im Bundestag: Je verbindlicher die Quotenforderungen, desto höher ist die Beteiligung und die konkrete Verschiebung zugunsten von Frauen in der jeweiligen Partei auf allen Ebenen, von innerparteilichen Ämtern bis hin zu den Parlamentssitzen.

\section{Stagnation - stößt die Quote an ihre Grenze?}

Die letzten beiden Bundestagswahlen haben keinerlei Verbesserung hinsichtlich der Vertretung von Frauen mehr herbeiführen können. Somit stellt sich die Frage, ob die Quote nach anfänglich großen Erfolgen - an ihre Grenzen gestoßen ist. Oder bleibt sie ein wirkungsvolles Instrument, das bisher lediglich nicht hinreichend ausgeschöpft wurde?

So ist es möglich, dass selbst jene Parteien, die Quotenregelungen haben, ihr Soll an weiblicher Repräsentanz in den Parlamenten nicht erfüllen, weil sie entweder Frauen auf ihren Wahllisten nicht entsprechend berücksichtigen oder Direktmandate weiterhin vorrangig an Männer vergeben. Allerdings könnte es auch der Fall sein, dass die Quote ihren Zweck durchaus erfüllt und die Parteien mit Quotenregelungen diesen auch gerecht werden, das Gesamtbild jedoch durch jene Parteien, die eine Quote weiterhin ablehnen, getrübt wird.

Um dies herauszufinden, empfiehlt sich zunächst ein Blick auf die Parteilisten für die Bundestagswahl 2009. Zwar haben die meisten Parteien, die über eine Quotenregelung verfügen, diese bei der Aufstellung der Kandidatenliste nicht erfüllt. Nur die CDU hatte mehr als die geforderte Anzahl an Listenplätzen an Frauen vergeben. Grüne und SPD hatten ihre Vorgaben leicht unterschritten, die Linke sogar deutlich. Da Frauen jedoch auf

9 Siehe dazu Global Database of Quotas for Women, http://www.quotaproject.org (Abruf am 1. August 2010). 
aussichtsreichen und letztendlich auch siegreichen Listenplätzen vertreten waren, konnten alle Parteien ihre Quotenanforderungen im Ergebnis übertreffen. Bei den beiden Parteien ohne Quotenregelung ${ }^{10}$ fallen die Ergebnisse deutlich negativer aus: Mit 32 beziehungsweise 23 Prozent weiblichen Listenkandidaten bilden CSU und FDP das Schlusslicht unter den im Bundestag vertretenen Parteien. Allerdings gilt es hier zu unterscheiden: Während die FDP ihre Abgeordneten ausschließlich über die Liste ins Parlament entsendet und damit den Frauenanteil gezielt steuern kann, ist dies bei der CSU nahezu unmöglich. Hier blieb die Liste - auf deren ersten zehn Plätzen immerhin fünf Frauen vertreten waren - wirkungslos, da die Partei die ihr zustehenden Bundestagssitze bereits mit Abgeordneten aus den Direktwahlkreisen ausfüllte und die Liste somit nicht zum Zuge kam.

\begin{tabular}{|l|c|c|c|c|c|c|}
\hline \multicolumn{7}{|l|}{ Tabelle 3: Frauenanteile auf den Parteilisten (in Prozent) } \\
\hline & CDU & CSU & FDP & SPD & Grüne & Linke \\
\hline Alle Listenplätze & 35 & 32 & 22 & 37 & 49 & 39 \\
Siegreiche Listenplätze & 43 & 0 & 26 & 43 & 58 & 56 \\
Quote & 33 & - & - & 40 & 50 & 50 \\
\hline Quelle: Louise K. Davidson-Schmich, Gender Quota Compliance and Contagion in the 2009 Bundestag \\
Election, in: German Politics and Society, 28. Jg. (2010), H. 3, S. 133-155, S. 142. \\
\hline
\end{tabular}

Während die Parteien mit Quotenregelungen also über die Listen ihren eigenen Anforderungen gerecht wurden, zeigt sich bei den Kandidaten und Gewinnern der Direktwahlkreise ein völlig anderes Bild: Keine einzige Partei konnte ihren Quotenanspruch hier erfüllen. ${ }^{11}$ Schon bei der Aufstellung der Bewerber für die Direktwahlkreise blieben alle Parteien hinter ihren eigenen Zielvorgaben zurück. Die Grünen und die Linke, die beide mindestens 50 Prozent Frauen auf ihren Listen fordern, haben nur 36 beziehungsweise 28 Prozent ihrer Direktwahlkreise an weibliche Kandidaten vergeben. Die SPD hat dies immerhin zu 36 Prozent getan und kam damit ihrer Quote von 40 Prozent deutlich näher. Die CDU vergab 22 Prozent ihrer Direktwahlkreise an Kandidatinnen und lag damit weit unter ihrem Quorum von 33 Prozent. Beide Parteien ohne Quotenregelung, CSU und FDP, hatten nur 13 beziehungsweise 16 Prozent Frauen unter ihren Wahlkreisbewerbern.

Für eine vertiefte Analyse ist es erforderlich, die Vergabe der Direktkandidaturen einer noch intensiveren Betrachtung zu unterziehen und zu unterscheiden zwischen Wahlkreisen, die für die jeweilige Partei als „sicher“, also mit hoher Wahrscheinlichkeit als siegreich gelten, und jenen, die als aussichtslos betrachtet werden. Als „sicher“ angenommen werden hier Wahlkreise, die ein Kandidat ein und derselben Partei bei den letzten beiden Bundestagswahlen 2002 und 2005 für sich entscheiden konnte, wobei das Ergebnis 2005 mindestens zehn Prozent Vorsprung vor dem Kandidaten einer anderen Partei betragen musste. Legt man diese Annahme zugrunde, konnten bei der Wahl 2009168 der 299 Direktmandate als „sicher“ betrachtet werden. ${ }^{12}$ Das bedeutet, dass dieselbe Anzahl an Sitzen als

10 Da die CSU den Beschluss zur Frauenquote erst auf ihrem Parteitag im Oktober 2010 fasste, wird sie in dieser Analyse noch als „Partei ohne Quotenregelung“ bezeichnet.

11 Allerdings sind Wahlkreise auch nicht quotiert.

12 Zwei dieser 168 Wahlkreise wurden 2009 neu abgegrenzt und werden deshalb in der Analyse nicht berücksichtigt. 
aussichtslos betrachtet werden kann, und zwar von jeder einzelnen Partei, die diese Sitze bei den letzten Wahlen nicht für sich entscheiden konnte. ${ }^{13}$

\begin{tabular}{|c|c|c|c|c|c|c|}
\hline \multicolumn{7}{|c|}{ Tabelle 4a: Anteil der Wablkreise, die 2009 an Frauen vergeben wurden (in Prozent) } \\
\hline & $\mathrm{CDU}$ & CSU & FDP & SPD & Grüne & Linke \\
\hline $\begin{array}{l}\text { an allen Direktmandaten } \\
(\mathrm{N}=299)\end{array}$ & 22 & 13 & 36 & 16 & 36 & 28 \\
\hline $\begin{array}{l}\text { an aussichtsreichen } \\
\text { Direktmandaten } \\
(\mathrm{N}=168)\end{array}$ & $\begin{array}{c}9 \\
(\mathrm{~N}=58)\end{array}$ & $\begin{array}{c}13 \\
(\mathrm{~N}=37)\end{array}$ & $\begin{array}{c}23 \\
(\mathrm{~N}=68)\end{array}$ & $\begin{array}{c}\text { k.A. } \\
(\mathrm{N}=0)\end{array}$ & $\begin{array}{c}0 \\
(\mathrm{~N}=1)\end{array}$ & $\begin{array}{c}100 \\
(\mathrm{~N}=2)\end{array}$ \\
\hline $\begin{array}{l}\text { an aussichtlosen Direkt- } \\
\text { mandaten }(\mathrm{N}=168)\end{array}$ & $\begin{array}{c}30 \\
(\mathrm{~N}=61)\end{array}$ & $\begin{array}{l}\text { k.A. } \\
(\mathrm{N}=0)\end{array}$ & $\begin{array}{c}40 \\
(\mathrm{~N}=79)\end{array}$ & $\begin{array}{l}\text { k.A. } \\
(\mathrm{N}=3)\end{array}$ & $\begin{array}{l}\text { k.A. } \\
(\mathrm{N}=2)\end{array}$ & $\begin{array}{c}52 \\
(\mathrm{~N}=21)\end{array}$ \\
\hline
\end{tabular}

\begin{tabular}{|l|c|c|c|c|c|c|}
\hline Tabelle 4b: Gewinner der Direktwablkreise 2009 (in Prozent) \\
\hline & CDU & CSU & FDP & SPD & Grüne & Linke \\
\hline Frauenanteil & 17 & 13 & 0 & 29 & 0 & 44 \\
Quote & 33 & - & - & 40 & 50 & 50 \\
\hline
\end{tabular}

Wie aus den Berechnungen hervorgeht, werden Frauen nicht nur seltener in Wahlkreisen als auf der Liste nominiert, sondern der Frauenanteil unter den Kandidaten verringert sich sogar noch deutlicher, wenn ein Wahlkreis als aussichtsreich gilt. Bei CDU und SPD ist dieser Unterschied besonders groß, mit gerade einmal neun beziehungsweise 23 Prozent Kandidatinnen in aussichtsreichen Wahlkreisen. Demgegenüber steht in beiden Parteien ein hoher Anteil an Kandidatinnen in aussichtslosen Wahlkreisen, nämlich 30 und 40 Prozent. Bei der CSU kann dies nicht nachgewiesen werden, da keiner ihrer Wahlkreise als aussichtslos gilt, der Frauenanteil unter den Direktkandidaten aber ohnehin nur 13 Prozent beträgt.

Zählt man die Abgeordneten der Listen und Direktwahlkreise zusammen, kommen die Parteien im 17. Bundestag auf die folgenden Anzahlen von Listen- und Direktmandaten:

\begin{tabular}{|l|c|c|c|c|c|c|}
\hline \multicolumn{7}{|l|}{ Tabelle 5: Verhältnis Direktmandate zu Listenmandate im 17. Deutschen Bundestag } \\
\hline Abgeordnete & CDU & CSU & FDP & SPD & Grüne & Linke \\
\hline $\begin{array}{l}\text { Listenplätze absolut (und } \\
\text { in Prozent) }\end{array}$ & $21(11 \%)$ & $0(0 \%)$ & $93(100 \%)$ & $82(56 \%)$ & $67(99 \%)$ & $60(79 \%)$ \\
$\begin{array}{l}\text { Direktwahlkreis absolut } \\
\text { (und in Prozent) }\end{array}$ & $173(89 \%)$ & $45(100 \%)$ & $0(0 \%)$ & $64(44 \%)$ & $1(1 \%)$ & $16(21 \%)$ \\
Frauenanteil (in Prozent) & 22 & 13 & 25 & 38 & 54 & 53 \\
Frauenquote (in Prozent) & 33 & - & - & 40 & 50 & 50 \\
\hline Quelle: Eigene Berechnung nach Angaben des Bundeswahlleiters zur Bundestagswahl 2009. \\
\hline
\end{tabular}

13 Tabelle 4a enthält jedoch nur Daten zu den Kandidaten und Kandidatinnen, die in den jeweiligen aussichtslosen Wahlkreisen von der Partei mit dem zweitgrößten Stimmanteil nominiert wurden. 
Aus Tabelle 5 geht sehr klar hervor, dass generell gilt: Die Kombination aus Quote und einer im Verhältnis zu den Direktmandaten großen Anzahl von Listenmandaten ist die beste Voraussetzung zur Steigerung des Frauenanteils. Die beiden Parteien mit dem höchsten Anteil an direkt gewählten Abgeordneten, CDU und CSU, verfügen mit 22 und 13 Prozent über die geringsten Frauenanteile im Bundestag. Bei SPD, Grünen und Linken zeigt sich hingegen, dass ein höherer Anteil an Listenplätzen einen höheren Frauenanteil zur Folge hat, und zwar umso deutlicher, je höher die jeweilige Quote angesetzt ist. Die FDP hingegen, die alle Abgeordneten über die Liste entsendet, aber über keine Frauenquote verfügt, hat zwar mehr Frauen als CDU und CSU, aber deutlich weniger als SPD, Grüne und Linke.

Würden die Mitglieder des Bundestages ausschließlich über Parteilisten gewählt, ergäbe sich - den aktuellen Stand hochgerechnet - ein Frauenanteil von 44 Prozent. Umgekehrt wären nur 22 Prozent der Bundestagsabgeordneten weiblich, würde sich das Parlament ausschließlich aus direkt Gewählten zusammensetzen.

\section{Die Kandidatenauswahl}

Wie aber lässt sich unter den gegebenen Voraussetzungen der Frauenanteil im Bundestag steigern? Nachdem die Möglichkeiten bei den Listenaufstellungen - mit Ausnahme der FDP - von allen Parteien ihren Vorgaben entsprechend ausgeschöpft werden, stellt sich insbesondere die Frage, ob und wie Direktmandate künftig stärker von Frauen besetzt werden können. Hier ist zunächst das Nominierungsverfahren genauer zu betrachten. Die Wissenschaftlichen Dienste des Bundestages beschreiben dieses Verfahren folgendermaßen: „Die tatsächliche Auswahl der Kandidaten für ein Wahlkreis-Direktmandat erfolgt in kleinen, informellen lokalen Parteizirkeln, die ihre Vorschläge den offiziellen Wahlgremien (Delegiertenversammlungen) präsentieren. Diesen Zirkeln gehören im Wesentlichen die Parteifunktionäre an, die über die tatsächliche Macht in den lokalen Parteiorganisationen verfügen. " 14

Verantwortlich für die Nominierung sind also die Führungsebenen auf Kreis- und Bezirksebene. In diesen informellen Netzwerken sind Frauen häufig stark unterrepräsentiert, weil ihr Anteil an innerparteilichen Führungspositionen gering ist. Besonders zu den in den drei großen Parteien zentralen Positionen der Kreisvorsitzenden erlangen Frauen nur schwer Zugang. ${ }^{15}$ Lediglich 26 Prozent der Kreis- und Unterbezirksvorsitzenden der SPD ${ }^{16}$ sind Frauen sowie 14 Prozent der CDU_-17 und neun Prozent der CSU_- ${ }^{18}$ Kreisvorsitzenden.

In einigen Ländern gibt es mittlerweile Versuche, neben den Listen auch auf die Wahlkreisbewerber Einfluss zu nehmen, doch sind die Widerstände an dieser Stelle besonders groß. Die Labour Party in Großbritannien verwendet beispielsweise „all-women-shortlists“,

14 Wilhelm Weege, Karrieren, Verhaltensmerkmale und Handlungsorientierungen von Bundestagsabgeordneten, Wissenschaftliche Dienste des Deutschen Bundestags WD 1 -069/03, Berlin 2003, S. 9.

15 Vgl. Heinrich-Böll-Stiftung, a.a.O. (Fn. 2), S. 20.

16 Zahlen: Gleichstellungsbericht der SPD.

17 Zahlen: Gleichstellungsbericht der CDU.

18 Zahlen nach Auskunft der CSU Mitgliederverwaltung. 
also Frauenlisten, aus denen für mindestens 50 Prozent der als sicher geltenden Wahlbezirke Direktkandidatinnen ausgewählt und aufgestellt werden müssen. Bei den Wahlen zum Schottischen Parlament und der Nationalversammlung von Wales wendete die Labour Party das „twinning“ Prinzip an, indem sie benachbarte Wahlkreise zu „Zwillingswahlkreisen“ machte, von denen jeweils einer mit einer Frau besetzt sein musste. ${ }^{19}$

\section{Die Zukunft der Frauenquote: Erfolgsrezept oder Auslaufmodell?}

Trotz der nachgewiesenen Wirksamkeit der Frauenquote wird immer wieder deutlich, dass diese durch unterschiedliche Gegebenheiten und Einflüsse teilweise stark eingeschränkt werden kann. Der Frauenanteil im Deutschen Bundestag stagniert seit fast zehn Jahren, und die vorausgegangene Analyse der Bundestagswahl 2009 zeigt, dass die Parteien ihre selbst gesteckten Ziele und Anforderungen nur teilweise erfüllen. Konkret stellt sich hier also die Frage, wie die Ausgestaltung und Umsetzung von Quotenregelungen in Zukunft wieder zu einer Steigerung des Frauenanteils in den Parlamenten führen kann.

Es gibt nach wie vor Bereiche, in denen die Quote ausgedehnt beziehungsweise konsequenter umgesetzt werden könnte, um ihre Wirksamkeit zu erhöhen. Die FDP lehnt das Instrument Frauenquote bis heute gänzlich ab, bei der CSU beschränken sich die neu eingeführten Vorgaben auf innerparteiliche Positionen auf Landes- und Bezirksebene. Würden diese Parteien eine wirkungsvolle Quote von 40 Prozent für Kandidaturen implementieren und sich auch daran halten, stiege der Frauenanteil im Bundestag auf 37 Prozent. ${ }^{20}$ Würde außerdem noch die CDU ihr Quorum strikter durchsetzen, läge der Anteil an weiblichen Abgeordneten bei 39 Prozent. Allerdings ist hier zu bedenken, dass die Landesebenen bei CDU und CSU aufgrund des hohen Anteils an Direktmandaten einen geringeren Einfluss auf die Zusammensetzung ihrer Fraktionen haben als die FDP, deren Vertreter ausschließlich über die Parteilisten der Landesverbände ins Parlament einziehen.

Da Frauen bei Union und SPD stärker auf den Listen als unter den Direktkandidaten zu finden sind, wirkt sich ein schlechtes Zweitstimmenergebnis im Verhältnis zu dem der Erststimmen immer negativ auf den Frauenanteil in der jeweiligen Fraktion aus. Somit kommt den Direktmandaten, die sich in Deutschland bisher jeglichen Quoten entziehen, eine Schlüsselposition zu. Um den Frauenanteil im Deutschen Bundestag zu steigern, bedarf es hier stärkerer Anstrengungen und gegebenenfalls auch deutlichere Vorgaben als bisher. Unbedingt notwendig ist zudem eine eingehende Analyse der innerparteilichen Führungsebenen auf Kreis-, Bezirks und Landesebene, die maßgeblichen Einfluss auf die Nominierung der Kandidaten nehmen. Gerade in den drei Parteien, die den größten Anteil an direkt gewählten Abgeordneten im Deutschen Bundestag stellen, CDU, CSU und SPD, nehmen Frauen bisher zu wenig einflussreiche Vorstandspositionen ein.

19 Vgl. Global Database of Quotas for Women, a.a.O. (Fn. 9).

20 Ausgehend vom Stand im Juli 2010 mit 622 Abgeordneten, davon 418 männlich und 204 weiblich. 